\title{
La oposición sindical en el Franquismo: Dispersión y territorialización de las fuentes
}

\author{
David RUIZ \\ (Universidad de Oviedo)
}

"Duro, largo y laborioso..." Así se caracterizaba en 1972, en un informe del Sindicato Vertical, el proceso que concluyó en la firma del primer convenio colectivo de Hunosa, la empresa pública ya entonces problemática de la minería asturiana. Términos estos que sin exageración pueden aplicarse hoy, veinte años después, a los escasos programas de recuperación y estudio de la documentación generada en aquél medio tan escasamente transparente, por no decir abiertamente hostil, y de tan prolongada duración.

Sin embargo preciso es señalar que no todas las carencias actuales en este plano sean achacables a la dictadura de Franco y a las adversas circunstancias de la clandestinidad y el exilio a las que fueron reducidos los trabajadores opositores del régimen. Porque al retraso en la constitución de los archivos sindicales con respecto al de los partidos de la oposición estatales (recuérdese que los del PSOE y del PCE se organizan entre 1977 y 1980, en cambio los de UGT y USO a comienzos de la década del ochenta y en el de Comisiones Obreras no se iniciarian los trabajos hasta muy al final de la misma), no será ajena la distinta suerte corrida por las centrales obreras con respecto a los partidos políticos durante la transición democrática. El indiscutible protagonismo alcanzado por estos últimos en la primera fase de la misma relegaría a los sindicatos, como no sin razones se reiteró en aquellos años, a la situación de "parientes pobres" de la transición en un momento en que las relaciones de correa de transmisión partido-sindicato aún permanecían en vigor, tanto en la órbita del socialismo como en la comunista.

Pese a todo, sin esperar a la apertura de los archivos sindicales, una exigua minoria de estudiosos pertinaces se dispondría desde el comienzo de los ochenta a investigar la condición laboral y la conflictividad obrera en la Dictadura por considerar que el estudio de estos fenómenos ofrecerían 
claves interpretativas equiparables al menos a las instancias políticas y partidarias para el conocimiento del régimen de Franco. Por mi parte, la triple circunstancia de encontrarme entre los seguidores de estas hipótesis, la de haber dirigido algunos estudios sobre el periodo la temática (dos Tesis Doctorales y otras dos Memorias de Licenciatura) y la de haber entrado finalmente en contacto con los trabajos preparatorios para la constitución del Archivo Histórico de Comisiones Obreras en la Fundación $1^{\circ}$ de Mayo, es la que justifica mi modesta aportación a estas Jornadas. Contribución breve que versará sobre la relación existente entre la actividad de los actores y la localización de los restos y las huellas, entre los programas y la acción política de las organizaciones y su legado documental, sin omitir finalmente la opinión sobre el mejor uso y administración de este patrimonio por considerar no sólo pertinente sino ineludible, la discusión sobre esta cuestión dada la convocatoria conjunta que hemos recibido archiveros e historiadores.

\section{LA PERIODIZACIÓN DE LAS RELACIONES LABORALES EN LA CRONOLOGIA DEL FRANQUISMO}

Hubo un tiempo, quizás excesivo, en que en el régimen de Franco en el plano académico pareció un coto reservado por y para economistas, los especializados en estructura particularmente. Con el apoyo de las estadísticas y otras fuentes oficiales, con ayuda de algún estudio sectorial y de miradas al pasado nacional y a la Europa Contemporánea, coincidieron en presentar la fecha de 1959 -la del cambio en la política económica que clausuraba la autarquía e iniciaba la liberalización-como el hito cronológico divisorio del régimen. De la seguridad de su elección (y del acierto, porque aquella decisión se revelará irreversible hasta hoy) dieron prueba la publicación de los primeros estudios con tendencia globalizadora antes aún de que la dictadura concluyese ${ }^{1}$. $Y$ de la notoria influencia de los economistas sobre la historiografía de la época, cierto es que ayudados por la coyuntura expansiva de los sesenta, rinde cuenta, por ejemplo, el hecho de que en la autoría del último volumen de la primera Historia de España que antes de la muerte de Franco incorporaba a ella la Dictadura (la dirigida por Miguel Artola) no figurase un historiador

El primer estudio sobre la primera fase de la historia económica del régimen fue obra de cinco autores catalanes (J. Clavera, J.M. Esteban, M.A. Monés A. Montserrat y J. RosHombravella) publicado en la editorial de la revista madrileña Cuadernos para el Diálogo (Edicusa) en 1973 con el título Capitalismo español: de la autarquia a la estabilización 1939-1959). 
contemporaneista, sino que fuese obra de un economista, de Ramón Tamames ${ }^{2}$.

Con leve retraso a los economistas, se produjo la incursión en la Dictadura de politólogos y sociólogos, especialmente atraídos por la incorporación de los tecnócratas a los gobiernos de Franco y por los efectos de los cambios económicos y de la modernización social de los sesenta. Fue este el período en el que autores como Juan José Linz, formado en la politología anglosajona, presentaron la dictadura del general Franco como un caso de régimen autoritario - los regímenes totalitarios continuarian definiendo a los del "socialismo real», una vez desaparecidos los fascistas italiano y alemán-, abriéndose desde entonces la aún inconclusa polémica sobre la naturaleza del régimen de Franco, justamente cuando declinaba la especulación esencialista sobre el nuevo caudillaje hispano alimentada por los teóricos de la Falange ${ }^{3}$.

Así pues, la ventaja inicial adquirida en el estudio de la dictadura por economistas, politólogos y sociólogos -la incorporación en tromba de estos se realizará a partir del último lustro del régimen a través de los Informes Foessa - no resultaba fácil de recuperar (ni entonces era posible) por los historiadores. Estos entonces debieron limitarse, en espera de la accesibilidad a fuentes solventes, a investigar sobre el periodo anterior a 1936 (mejor aún, con mayores garantías, a etapas anteriores a 1931), a leer las publicaciones de los hispanistas arriesgados, las de economistas y sociólogos, y a esperar tiempos mejores. $Y$ a escuchar también, en alguno de los raros foros de especialistas dedicado a la metodología, intervenciones a favor de los derechos del historiador bajo la Dictadura. Desde 1968 resultó habitual la queja de investigadores sobre la limitación de fuentes, así como la denuncia por las discriminaciones principalmente en la cita anual de los coloquios de Pau (Francia). En el interior, sin embargo, habrá que esperar a 1972 para oír la valiente intervención del historiador francés Ernest Labrousse a favor de las libertades de expresión y de investigación histórica en el discurso de clausura del Congreso Internacional celebrado aquél año en Santiago de Compostela, en presencia de las autoridades académicas y políticas franquistas entre los que se encontraba el medievalista Luis Suárez Fernández, entonces director general de Universidades y actualmente en la presidencia de la Fundación Francisco Franco.

\footnotetext{
Se publicó en 1973 en Madrid por Alianza Editorial con el título La República. La Era de Franco (1931-1970)

3 En 1970, el citado articulo ("An authoritarian regime: the case of Spainn) fue incluido por Allardt y Rokkan (eds) en el volumen Mass politics: Studies in political sociology. New York, Free Press.
} 
En todo caso la espera sería menor para los historiadores interesados en la oposición política (más breve aún para los de la esfera del pensamiento) que para los estudiosos de la oposición sindical, por los motivos a los que anteriormente aludimos. No obstante la importancia creciente que durante la transición adquirieron las relaciones laborales, la centralidad que durante la crisis desempeñaron mecanismos como la negociación concertada y los grandes pactos, llevaron a revalorizar la Ley de Convenios Colectivos de 1958 como hito sociolaboral equiparable a 1959 en el plano económico $-\mathrm{y}$ a la crisis de 1956 en el plano más estrictamente político- en la periodización del régimen de Franco.

Así mismo, desde la perspectiva de 1958 cobra más sentido la publicación veinte años atrás del Fuero del Trabajo (marzo de 1938) con el que se abre el nuevo orden en este plano, caracterizado por la intervención estatal y el reglamentismo laboral, además de sentar las bases del Sindicato Vertical sobre el que, como es sabido, se ejercerá primordialmente, la oposición sindical. Y ello porque ante la publicación por la Dictadura naciente del Fuero del Trabajo, palidecerán otras decisiones del régimen que ocasionalmente, sin embargo, no se les ha escatimado relieve como fueron la celebración de las primeras elecciones sindicales (1944) o la constitución de los jurados de empresa (1953), probablemente las únicas efemérides reseñables junto a la normativa de los Convenios en la historia de las relaciones laborales. La rareza de episodios reseñables en esta esfera afectó al Sindicato Vertical ya que apenas ofrece otro cambio que el registrado en 1941 cuando dos años después de nombrado era destituido Gerardo Salvador Merino, primer Delegado Nacional de Sindicatos, el falangista "puro" que aspiró a dotar a aquél importante aparato de un grado de autonomía insoportable entonces para el Caudillo y su entorno de poder.

La conflictividad en la esfera industrial (no en la agraria) será lógicamente la referencia única que la oposición aportará a la periodización. No obstante conviene retener la fecha de 1963 por concluir en ese año la etapa pre-estadística de la Dictadura que, en este plano, remonta sus origenes a 1936. Pero si precisa es la referencia a fuentes oficiales del Ministerio de Trabajo a partir de 1963, más ineludible es la búsqueda de fuentes de procedencia más diversa para los años 1939-1963; período este en el que el férreo control al que se vieron sometidos la inmensa mayoría de los trabajadores no impedirá sin embargo la aparición de resistencias y rechazos a diferentes escalas: individual, de grupo, de taller, de fábrica, de sector laboral, incluso de localidad. Actitudes en general registradas como algo excepcional a la predominante aceptación pasiva y masiva de las condiciones laborales impuestas por los vencedores de la guerra civil. 
Finalmente una última reflexión sobre la cronología de la oposición. Con escasa antelación a la publicación de las estadísticas de huelgas por el Ministerio de Trabajo, en 1962 se registrará una movilización obrera en la minería asturiana con importantes repercusiones en otras zonas laborales de España y resonancias en los medios de comunicación internacionales, llegando a ser presentado el conflicto por el conjunto de la oposición como «el principio del fin de la Dictadura", en unos casos o como el más aséptico, "comienzo de una nueva etapa», en otros. En cualquier caso, a más de treinta años de distancia, hoy es francamente difícil con las fuentes disponibles argumentar en contra de las apreciaciones emitidas por los más lúcidos contemporáneos del acontecimiento, pasando la huelga de 1962 a constituir, en cierto modo, la réplica a la referencia dominante, a la del poder, de 1959.

$Y$ ello sobre todo porque los datos posteriores la avalaron: con la huelga minera de 1962 se inicia un nuevo ciclo conflictivo que tras veinticinco años concluirá en 1977 - fecha de la disolución del Sindicato Vertical y legalización de los sindicatos de clase- al que se incorporan los nuevos trabajadores de las áreas industrializadas en los sesenta. Ciclo en el que, según las estadísticas del Ministerio de Trabajo, el año 1970 registrará el más elevado número de conflictos (1.595) y 1974 el mayor número de trabajadores en huelga (625.971).

\section{LA DIVERSIDAD DE PROCEDENCIA DE LOS FONDOS DOCUMENTALES DE LOS SINDICATOS DE LA OPOSICIÓN}

Al concluir el régimen de Franco se tuvo la ocasión de percibir, por un momento, que la persecución y el elevado nivel destructivo de la Dictadura sobre las organizaciones sindicales de clase no había logrado erradicar, sin embargo, la aspiración ya secular y recurrente a la unidad orgánica de los trabajadores. Pero, una vez más, el proyecto unitario impulsado entonces desde el interior por las Comisiones Obreras no se cumplió; la pluralidad sindical triunfó, y la división entre centrales históricas y las de reciente formación, de nuevo adquiría carta de naturaleza en los comienzos de la transición democrática. Los archivos sindicales, en consecuencia, al mismo tiempo que significaban una atractiva novedad historiográfica se constituyeron separadamente.

$Y$ de este modo, tal como es conocido, la Fundación Largo Caballero se convertiria en la principal depositaria del Archivo de la UGT desde el comienzo de la transición, según se informaba en la XV reunión de la IALHI (Internacional Asociation of Labour History Institutions). Un archivo 
en el que tanto el volumen como el contenido de la masa documental no podrá menos que reflejar la estrategia del sindicato en la Dictadura y evidenciar, en consecuencia, el desequilibrio de los fondos al proceder en su mayoria de las organizaciones del exilio ${ }^{4}$.

Mas problemático ha sido el proceso de recuperación archivístico de la CNT pese a beneficiarse de la oportunidad que en este plano también ofreció el largo exilio. Sin embargo los siempre negativos efectos de las escisiones orgánicas sobre el patrimonio documental, a pesar de no haber cristalizado hasta el reciente período de la democracia, han determinado que de la custodia de los fondos principales de la histórica Confederación Sindical se haya responsabilizado el Instituto de Historia Social de Amsterdam. Fondos en los que el desigual contenido de los procedentes de la clandestinidad y del exilio probablemente revista una proporción similar al existente en los archivos de la UGT ${ }^{5}$.

De muy distinto signo fueron las experiencias sindicales opositoras desarrolladas en el interior de forma independiente a las centrales históricas. Las diferencias con respecto a estas radicaron, como es conocido, en la diferente estrategia y en la filiación ideológica de las dos corrientes predominantes, la católica y la comunista. Ambas, mientras no se pruebe lo contrario, inician su actividad a fines de los años cuarenta: en 1948 tienen lugar las primeras jornadas nacionales de la JOAC (JOC desde 1956) y en esa misma fecha se registra la recomendación de Stalin a la dirección del PCE de la conveniencia del viraje en la lucha antifranquista, es decir, del abandono de la guerrilla y la utilización opositora de la plataformas legales del franquismo. No obstante, en los dos casos resulta difícil aún precisar el grado de infiltración en el Vertical antes de mediados de los cincuenta. En todo caso en los fondos de la JOC, según datos relativamente recientes,

4 Herminia Arrazola Moreno “La experiencia de la Fundación Largo Caballero. Una alternativa para los archivos sindicales" en Archivos para la historia del movimiento obrero español (XV conferencia de la IALHI, 1984) Madrid, Edit. Pablo Iglesias, 1985; pp. 67-73 y “Archivo de la UGT de España en el exilio. Fundación Largo Caballero" en La oposición al régimen de Franco. Madrid, UNED, 1990; pp. 435-447.

Sobre los fondos de la UGT en la Fundación Pablo Iglesias, Aurelio Martín Nájera, «Fuentes archivisticas para la historia del socialismo español: clandestinidad y exilio" en La oposición al régimen de Franco, ob.cit. pp.473-485.

5 Pese a reiterados intentos cerca de la Fundación Salvador Seguí de Madrid, nos ha sido imposible verificar en qué medida se ha cumplido el plan presentado en 1984 por José Ramón Palacios Garcia, en la XV Conferencia de la IALHI, de depositar en una Fundación vinculada a la CNT el bloque documental de la posguerra, hasta la detención del último Comité Nacional en el interior (1939-1959). Ver "Génesis problemática de una Fundación de Estudios Libertarios" en Archivos para la historia del movimiento obrero español. ob. cit., pp. 35-39. 
existe documentación sobre el movimiento obrero y juvenil desde aquellas fechas ${ }^{6}$.

No obstante los precedentes del movimiento apostólico seglar, la oposición sindical de mayor entidad que se reclama de esta procedencia la ejercerá la Unión Sindical Obrera fundada en 1960. Tanto bajo la dictadura como en la posterior transición democrática, la USO se presentará como una organización de clase de inspiración cristiana, pero aconfesional y sin referente partidario colaborando en la oposición con las Comisiones Obreras en los primeros sesenta; posteriormente sufrió algunas escisiones y se afirmó en la defensa de los valores humanistas y socialistas ${ }^{7}$.

Las Comisiones Obreras, en cambio, resultado de la coordinación de grupos laboral y geográficamente dispersos de oposición surgidos de forma semi-espontánea en los años cincuenta, primeramente apoyados por el PCE y más tarde también por la constelación de grupos situados a la izquierda de éste, se autodefinirán en los primeros sesenta como movimiento reivindicativo, sociopolítico, democrático y de clase, hasta su transformación en Confederación Sindical a finales de 1976.

No puede dejar de reseñarse que a los efectos documentales tanto las fuentes de la USO como las de Comisiones Obreras adolecerán de las carencias inherentes a sus origenes y práctica política con respecto a las centrales históricas. No dispondrán, en especial la USO, de infraestructura archivística en el exterior como alternativa a los riesgos que entramaba la custodia de archivos centralizados en el interior en la situación de clandestinidad. Factor este al que se añadirán otros como la ausencia de la necesaria burocracia y el inmediatismo que en el interior caracterizó a la lucha sindical, más aún que a la política de los partidos clandestinos.

Por lo que respecta a las Comisiones Obreras, tanto las diferencias de origen con el resto de las centrales sindicales, incluida la USO, como el carácter de movimiento que adoptaron las Comisiones y la impresión que dieron sus promotores de mantenerlas en una tensión tan volcada hacia el futuro que muchos creyeron poderse permitir la licencia de prescindir del pasado..., se acusarán en la constitución de su archivo histórico ${ }^{8}$.

\footnotetext{
- Salvador Segundo Serrano, Guia Inventario del Archivo de la Juventud Obrera Cristiana (JOC). Madrid, Ministerio de Cultura, 1987.

7 Ver art. de A. Martín Artiles, «Del blindaje de la sotana al sindicalismo aconfesional. Breve introducción a la historia de la Unión Sindical Obrera, 1960-1975" en La oposición al régimen de Franco, ob.cit. pp. 165-168.

\& A algunos problemas y rasgos diferenciales he aludido recientemente en la presentación del volumen Demandas obreras y tribunales franquistas, primer Catálogo documental de la Fundación $1^{\circ}$ de mayo, recientemente publicado (Madrid, 1991).
} 
Afortunadamente, sin embargo, aquella especie de nuevo mesianismo que afectó a un sector del antifranquismo, dejó más restos documentales de los inicialmente previsibles, como recientemente se viene comprobando desde la Fundación $1^{\circ}$ de Mayo de Madrid. Porque además de la documentación recuperada sobre y de las Comisiones Obreras existente en el Archivo del Partido Comunista de España, en el Archivo Histórico Nacional y en el General de la Administración Civil, se preservó, por ejemplo, el fondo reunido en la Delegación Exterior del propio movimiento de oposición sindical instalada en París desde 1968, la DECO. A otros niveles pertenecen, así mismo, las fuentes preservadas por un número significativo de militantes de diversos sectores laborales y regionales de la organización.

\section{LA TERRITORIALIZACIÓN}

A diferencia de la actividad de los partidos y otros grupos menos organizados que actuaron en las grandes ciudades, es sabido que la oposición sindical nació y creció en las fábricas, en las de mayor tamaño antes que los medianos y pequeños, asociada a las obligaciones laborales antes que a la disciplina de los militantes partidarios de la clandestinidad.

El itinerario geográfico seguido por la protesta laboral hasta ahora conocida nos muestra, sin embargo, cómo en las primeras décadas del franquismo la principal actividad no se registró en grandes núcleos urbanos, sino en comarcas como la del Vallés en Cataluña, la margen izquierda de la ria del Nervión en el País Vasco, las cuencas mineras en Asturias, o el Marco de Jeréz en Andalucia, por señalar sólo las cuatro zonas más significativas. Pero es que después, a partir de los sesenta se incorporarán a las nuevas industrias una población laboral, en su mayoría procedente de la emigración, que se instalaría en cinturones, ensanches o polígonos y que, en general, rebasaron el ámbito tradicional urbano modificando la fisonomía del barrio obrero clásico, del conformado antes de 1936.

Por otra parte la dinámica de la conflictividad y de la represión —sobre todo la de aquella, determinada por ésta- también contribuirá durante décadas a que, a diferencia del pasado, no se desarrolle en las calles sino en los centros de trabajo y en los lugares vivienda más próximos a ellos. Porque los trabajadores actúan principalmente en ambos escenarios. En ellos cumplen la jornada laboral, votan o hacen el boicot en las elecciones sindicales, se reúnen los enlaces y jurados de empresa, agitan los grupos o comisiones de fábrica, se constituyen las coordinadoras comarcales y locales (en unos casos en las parroquias, en otros en los locales del 
Vertical), discuten las reivindicaciones, organizan los paros, etc. Sólo excepcionalmente, y en períodos muy tardíos, la conflictividad obrera durante la Dictadura se manifestará externamente haciendo acto de presencia en los núcleos urbanos, en los centros de las capitales de provincia y del Estado.

Asi pues, al margen de la indiscutible importancia de los archivos policiales, empresariales y la de los propios órganos representativos del sindicato o movimiento, lo que tratamos de significar es que bajo un régimen de privación de libertades como fue el del General Franco, la actuación de los trabajadores asalariados se redujo a los territorios fabril y vecinal, siendo estos ámbitos los generadores primordiales de la documentación escrita y oral de la oposición activa al Sindicato Vertical. De ahí el interés complementario que pueden adquirir las fuentes para el estudio de los asentamientos migratorios como elemento integrante de manifestaciones de rechazo al orden vigente en otros niveles no estrictamente laborales, como la protesta del vecindario urbano en el tramo final de la Dictadura.

Por lo esquemáticamente expuesto conviene preguntarse sobre la relación y los efectos de la territorialización de estas fuentes y archivos sindicales. En lo concerniente a las Comisiones Obreras, la coordinación desde la Fundación $1^{\circ}$ de Mayo de los trabajos preparatorios orientados a la constitución del Archivo Histórico de la citada Confederación permite resaltar las siguientes consideraciones:

1. Q Que la conciencia territorial de los actores del pasado de las Comisiones Obreras se ha transferido, en gran medida, a la recuperación y custodia del patrimonio documental. Y que este, a diferencia del de la UGT y probablemente del de la CNT y de la USO, no fue generado principalmente por los órganos de dirección y sí, en cambio, por los escalones intermedios del movimiento opositor.

2. a Que la territorialidad incipiente en los cincuenta y desarrollada en los sesenta, tendió a solaparse con la protesta política regional de los primeros setenta. Importante factor este, cuando al comienzo de la transición, el movimiento de las Comisiones Obreras se transforme en Confederación Sindical, primándose la estructura territorial sobre la federal de la organización.

3. ${ }^{-}$Que la regionalización de la estructura del nuevo Sindicato adaptado (con la excepción de Navarra) al de las Comunidades del Estado de las Autonomías, se advertirá en la fase de coordinación del movimiento según delatan los comunicados sucesivos realizados desde Madrid a mediados de los sesenta. Antes de que el término Estatal figurase en ellos, los dirigentes más representativos de las regiones pasarian de participar en la Asamblea Nacional, en junio de 1967, a las Reuniones y Coordinadoras 
Generales desde diciembre del mismo año hasta el final de la Dictadura. La presencia de las Comisiones Obreras catalanas y vascas no fueron, lógicamente, ajenas a los cambios en los términos como tampoco dejó de apreciarse la relación de las Comisiones Obreras con nacionalismos de menor vigor que el catalán y el vasco, como lo prueba el que las Comisiones Obreras levantinas se constituyeran en la sede de Lo rat penat de Valencia en 1966.

Asi pues el lento pero progresivo arraigo de la territorialidad y no las donaciones de fondos documentales ni tampoco un deliberado proyecto de "federación", explica la apertura de diversos centros de depósito, ordenación e inventariado en las capitales de las Comunidades Autónomas (en diez de ellas hasta el presente) siendo coordinado el trabajo desde la Fundación $1^{\circ}$ de Mayo de Madrid y supervisado hasta hoy por la Dirección General de Archivos Estatales.

Distinto proceso, pues, el seguido por las Comisiones Obreras que la suerte corrida por la Unión Sindical Obrera, organización afectada por una fuerte escisión en los comienzos de la transición que dió lugar al ingreso en la UGT de un sector de la misma con importante peso en él del grupo madrileño. Circunstancia esta que ayuda a entender cómo el Archivo del sindicato que nacerá en 1960 en Rentería (Guipúzcoa) acabó instalándose en 1983 en Martorell (Barcelona). El hecho se debió, en palabras del actual director de la Universidad Sindical de USO enclavada en la citada localidad catalana, a la integración en la UGT, en 1977, del sector madrileño de los colaboradores del Centro de Estudies Sociales creado por la USO, opción que no fue secundada por el grupo barcelonés ${ }^{9}$. Escisión sindical que, como frecuentemente ocurre en estos trances, también afectará a parte del fondo documental, habiéndose depositado en la Fundación Pablo Iglesias el archivo de la Federación de Banca de la USO y en la Largo Caballero el de José Maria Zufiaur, anterior dirigente del mismo sindicato ${ }^{10}$.

Llegados a este punto conviene reflexionar brevemente sobre las actitudes de los investigadores ante la dispersión actualmente existente de los archivos sindicales y del movimiento obrero. La impresión dominante es que el policentrismo documental continuará acentuándose como lo prueba el hecho de que incluso sindicatos tan reacios a la descentralización como es el caso de la UGT, cuente actualmente con centros de documentación

\footnotetext{
9 Información para esta ponencia que debo a la cortesia de Antonio Martín Artiles, director de la Universidad Sindical de Martorell.

10 Ver articulos de $\mathrm{H}$. Arrazola y A. Martín en La oposición al régimen de Franco, cit.
} 
abiertos en Cataluña (Fundación Rafael Campalans, Barcelona) en Asturias (Fundación José Barreiro, Oviedo) y en Galicia (Fundación Luis Tilve, Santiago de Compostela). Y que el fenómeno, además, no parece encontrar hasta ahora resistencias notorias entre los estudiosos e investigadores.

La explicación probablemente proceda del crecimiento de la historiografía regional, tendencia que prendió en nuestro país hace ya un cuarto de siglo y que no parece dar síntomas de debilitamiento. Es más, al margen de consideraciones teóricas y de método, la coyuntura política que se inició con la protesta regionalista bajo la Dictadura y se plasmó constitucionalmente en 1978 en el nuevo Estado de las Autonomias, le ha sido francamente favorable.

En cuanto a la temática del sindicalismo y la oposición en la época de Franco, el contencioso que se viene manteniendo en la última década entre la demanda investigadora y la oferta archivística, ya no tiene para los primeros como única referencia las instancias centrales. La territorialización de los centros documentales, en general, satisface a los nuevos investigadores, refuerza líneas de investigación en los departamentos universitarios, estimula el interés y, por lo que parece, facilita la recuperación de fuentes.

Esta fue, al menos, la idea más compartida entre un grupo de investigadores, algunos más experimentados y otros más recientes que se iniciaban en estas lides, a lo largo de un Seminario que sobre "Condición laboral, conflictividad y oposición sindical al franquismo» en los siete principales territorios peninsulares, se celebró en la Fundación $1^{\circ}$ de Mayo durante el curso 1990-1991. Un Seminario en el que la información de los ponentes sobre el estado de la investigación en las diferentes escalas territoriales: regional, comarcal, local y de fábrica, suscitó un nivel de aceptación inversamente proporcional, se diría, al estado en que se encuentran las fuentes.

- Sin embargo, en este último capítulo, una vez más, se tuvo la oportunidad de conocer nuevos datos sobre pérdidas, destrucción y resistencias a la entrega de documentos, sin excluir casos de apropiaciones indebidas, etc. Tampoco podía faltar información sobre las diferencias de comportamientos "territoriales" en relación con los demandantes, con los nuevos usuarios de los Archivos Provinciales. Los fondos de la AISS precisamente figuraron como el ejemplo más socorrido y reiterado de la accesibilidad, en unos casos, y de la prohibición en otros; o la diversidad de situaciones en los Archivos relacionados con documentación procedente de los gobiernos civiles franquistas describiéndose hasta tres modalidades de relación con 
los usuarios: la no accesibilidad, la de tolerar la consulta pero no la toma de notas, y la consulta regular sin restricciones.

En fin, una situación que aún requiere de remedios eficaces para reducir la dureza adicional de esta especialidad investigadora con respecto a la clásica del oficio, problema al que aludíamos al comienzo de la ponencia. Dificultades circunstancialmente compensadas, únicamente, con la recuperación de fondos documentales imprevistos y el descubrimiento de nuevos órganos de procedencia. Los problemas, sin embargo, persistirán mientras no se establezca una mayor comunicación entre la administración y los administrados, entre los archiveros y los investigadores en este caso. Porque una comunicación fluida, normalizada, entre ambos estamentos, favorecería la elemental pero precisa formación archivística de los historiadores a la vez que permitiría la actualización historiográfica de los archiveros. Creo que era la intención de estas Jornadas a las que fuimos convocados. Y para terminar, una propuesta. Dado que la recuperada libertad sindical ha venido asociada a la pluralidad de organizaciones y dado lugar a la diversidad de centros de documentación (con las ventajas pero también los inconvenientes funcionales que ello plantea y que todos conocemos), no parece descabellado abordar la perspectiva de iniciar un proceso de centralización. Al menos no lo era años atrás para el ministro de Cultura Jorge Semprún quien barajó la idea de un archivo del sindicalismo y movimiento obrero adscrito al Histórico Nacional, según la fórmula de la Sección Guerra Civil de Salamanca. Un proyecto que lógicamente garantizaría la legitimidad de la propiedad y los derechos de las partes afectadas y que, de llevarse a buen fin, además de incentivar la investigación, eliminaría los consabidos riesgos al patrimonio documental de los trabajadores asalariados del país y de sus organizaciones cuya importancia ya no precisa subrayarse. 